\title{
Treatment of Irritable Bowel Syndrome with a Combination of Curcumin, Green Tea and Selenomethionine Has a Positive Effect on Satisfaction with Bowel Habits
}

\author{
0. Lior, F. Sklerovsy-Benjaminov, I. Lish, A. Stein, F. Konokoff, T. Naftali \\ Meir Medical Center, Kfar Saba, Israel \\ Email: Timna.naftali@clalit.org.il
}

How to cite this paper: Lior, O., Sklerovsy-Benjaminov, F., Lish, I., Stein, A., Konokoff, F. and Naftali, T. (2019) Treatment of Irritable Bowel Syndrome with a Combination of Curcumin, Green Tea and Selenomethionine Has a Positive Effect on Satisfaction with Bowel Habits. Journal of Biosciences and Medicines, 7, 170-179. https://doi.org/10.4236/jbm.2019.75018

Received: February 19, 2019

Accepted: May 24, 2019

Published: May 27, 2019

Copyright $\odot 2019$ by author(s) and Scientific Research Publishing Inc. This work is licensed under the Creative Commons Attribution International License (CC BY 4.0).

http://creativecommons.org/licenses/by/4.0/

\begin{abstract}
Background: Irritable bowel syndrome (IBS) is a common disorder impairing patient's quality of life. Currently, there is no effective treatment for this syndrome. Coltect is a dietary supplement containing curcumin, green tea and selenomethionine. Aim: To investigate the effects of Coltect on IBS symptoms. Methods: A randomized, prospective, placebo-controlled, double blinded, crossover study. The study population included patients diagnosed with IBS by Rome criteria. Subjects were randomized to receive either Coltect or placebo for 4 weeks, and then received the opposite treatment for 4 weeks following a washout period of 2 weeks. The placebo was a pill, identical in color and size to the Coltect pill, with no active ingredients. Patients completed IBS health-related quality of life, severity score and IBS symptom questionnaires before and after each treatment period. Results. The study included 22 patients, ages $22-77$ years. Coltect had a positive effect on patients' satisfaction with their bowel habits (as measured on a scale from 0 -Very satisfied to $100-$ Very unsatisfied). Average score was $70.4 \pm 33.1$ before and $61.8 \pm 29.3$ after Coltect treatment $(\mathrm{p}=0.037)$. Other parameters, including abdominal pain, bloating, and effects of IBS on daily activity, symptoms, IBS-related quality of life and general health quality of life were unchanged. Conclusion: Coltect seems to have a positive effect on satisfaction with bowel habits.
\end{abstract}

\section{Keywords}

Irritable Bowel Syndrome, Coltect, Curcumin, Selenium, Green Tea, IBS Health Related Quality of Life Questionnaire, Severity Score Questionnaire, IBS Symptom Questionnaire, Bowel Habits 


\section{Introduction}

Irritable bowel syndrome (IBS) is a common functional gastrointestinal disorder. Primary symptoms are abdominal pain, bloating and altered bowel habits in the absence of identified organic abnormalities. Quality of life is often affected in IBS patients, leading to poor work productivity and excessive health care utilization [1] [2].

Since IBD is defined by clinical symptoms, diagnostic criteria known as the Rome criteria have been established [3]. According to the Rome III Criteria, IBS is defined as recurrent abdominal pain, at least one day per week in the last three months, associated with two or more of the following:

- Related to defecation;

- Associated with a change in stool frequency;

- Associated with a change in stool form (appearance).

Subtypes of IBS are recognized based on patient-reported predominant patterns of bowel habits on days with abnormal bowel movements. IBS subtypes are defined for clinical practice as follows:

- IBS with predominant constipation: Patient reports that abnormal bowel movements are usually constipation;

- IBS with diarrhea: Patient reports that abnormal bowel movements are usually diarrhea;

- Mixed IBS: Patient reports that abnormal bowel movements alternate between constipation and diarrhea (more than one-fourth of all the abnormal bowel movements are constipation and more than one-fourth are diarrhea);

- Unclassified IBS: Patients who meet diagnostic criteria for IBS but cannot be accurately categorized into one of the three subtypes.

Treatment options might include non-medical approaches such as dietary modifications, change in lifestyle and cognitive behavior therapy [4] medical approaches include drugs such as laxatives, lubiproston [5] linaclotide, antidiarrheals, alosetron, rifaximin [6], and eluxadoline [7].

Additional therapies including herbal products have been evaluated in IBS patients. However, most current treatment options were effective for a limited number of patients and many were not validated in randomized, controlled studies.

Evidence supports low grade inflammation as the inciting factor in IBS, leading to visceral hypersensitivity and alteration in bowel motility in IBS. Immunohistologic investigation has revealed mucosal immune system activation, characterized by alterations in particular immune cells and markers in some patients with IBS [8]. [9] Increased number of lymphocytes has been reported in the colon and small intestine in patients with IBS. These cells release mediators (nitric oxide, histamine and proteases) capable of stimulating the enteric nervous system, leading to abnormal motor and visceral responses within the intestine [10]. Stool examinations from diarrhea-predominant IBS patients revealed a high level of serine protease activity [11] [12]. An increased number 
of mast cells have been demonstrated in the terminal ileum, jejunum and colon of IBS patients [13]. Studies have demonstrated a correlation between abdominal pain in IBS and the presence of activated mast cells in proximity to Colonicnerves [10]. Moreover, elevated levels of plasma pro-inflammatory interleukins have been observed in patients with IBS [14]. In addition, peripheral blood mononuclear cells of IBS patients produce higher amounts of tumor necrosis factor than do healthy controls) [15] [16].

Coltect (NavehPharma, Ltd., Netanya, Israel) is a dietary supplement provided in oral tablets containing $500 \mathrm{mg}$ curcumin, $250 \mathrm{mg}$ green tea and $100 \mu \mathrm{g}$ selenomethionine. It has been associated with positive effects on inflammatory diseases such as ulcerative colitis [15]. Coltect is approved as a food supplement by the Ministry of Health in Israel.

Curcumin is a natural compound found in the plant Curcuma longa. It is also known as turmeric and used as a food additive. The primarying redient in turmeric is curcumin (chemical name diferuloylmethane), which has both anti-inflammatory and antioxidant properties [15] [16]. Curcumin inhibits mediators of inflammation such as NFkB [17], cyclooxygenase-2 (COX-2), lipoxygenase (LOX) [18], IL-2 and inducible nitric oxide synthase (iNOS).

Green tea is made from unfermented leaves of Camellia sinensis and contains a very high concentration of polyphenols, which are powerful antioxidants. Green tea polyphenols include epigallocatechin gallate (EGCG), epigallocatechin (EGC), epicatechin gallate (ECG) and epicatechin (EC). A study in an animal model of inflammation-related colitis, provides evidence that green tea polyphenols have anti-inflammatory effects on murine colitis [19].

Selenium is an essential trace element. The average nutritional intake worldwide is 50 to $350 \mu \mathrm{g} / \mathrm{day}$. Selenium is important for thyroid and cardiovascular function and selenium deficiency is associated with increased morbidity in HIV patients [20]. Selenium is an antioxidant and anti-inflammatory agent and is associated with colorectal cancer prevention [21]. Low levels of selenium were associated with active inflammation in patients with inflammatory arthritis and were increased after anti-inflammatory treatment [21].

A clinical study with Coltect found beneficial effects in patients with mild to moderate ulcerative colitis [22]. Based on the anti-inflammatory and antioxidant properties of the various ingredients of Coltect, we hypothesized that it would have the potential to improve symptoms in patients with IBS. The current study investigated the efficacy of Coltect in improving symptoms of IBS, in a prospective randomized, placebo-controlled, double blinded, crossover design.

The aim of the study was to investigate the effect of a combination of three natural ingredients: Curcumin, Selenomethionine and green tea, on symptoms of patients with irritable bowel syndrome.

\section{Materials and Methods}

This prospective, double blind, placebo-controlled, crossover study was con- 
ducted from 2011 to 2017. The investigation was approved by the Ethics Committee of Meir Medical Center. All patients signed informed consent prior to participating in the study.

\subsection{Population}

Patients with a diagnosis of IBS according to the Rome III criteria who attended the Gastroenterology Clinic at Meir Medical Center in Israel were recruited to the study participants were interviewed by a physician who assessed the diagnosis of IBS according to the Rome III criteria, and completed the study questionnaires.

Inclusion criteria were: ages 18 to 80 years, gastrointestinal examination in the past 5 years, no treatment changes at least 2 weeks before the study or during the study, and no antibiotic treatment at least 2 weeks before the study. Patients with a previous major abdominal surgery, known gastrointestinal disorder (such as IBD, celiac, carcinoma or absorption disorder), psychiatric disorder, and other major medical conditions such as severe congestive heart failure, severe diabetes mellitus, renal failure, or cirrhosis were excluded.

Patients were randomized to either treatment first or placebo first arms, using the block method in a 1:1 ratio [23].

\subsection{Protocol}

Disease severity was assessed using irritable bowel severity score (IBSS) which utilizes a visual analog scale (VAS) the IBS quality of life (QOL) questionnaire [24] and health-related QOL questionnaires [25]. IBS symptoms were assessed using a specific, quantified scale, with a scoring system from 1 (mild symptoms) to 5 (severe symptoms), grading 8 specific IBS symptoms, including abdominal discomfort, abdominal pain, bloating, gas, mucous discharge, constipation, diarrhea, and alternating constipation and diarrhea. The questionnaires included multiple choice questions regarding the symptoms of IBS, and were scored on a scale from 1 to 5 points (1-No symptoms, 5-Severe symptoms). A fecal culture was obtained to rule out an infectious cause of gastrointestinal symptoms. Participants were randomly assigned to receive Coltectata dose of 4 tablets/day, (courtesy of Nave pharma, Netania, Israel) or a placebo containing no active ingredients, for 4 weeks. They then entered a 2-week washout period during which they did not receive the study medication. This was followed by a crossover treatment arm for another 4 weeks.

\section{Statistical Analysis}

When research was defined, we assumed 20\% improvement between patient's status in the beginning and end of the study. Minimum sample size expected was 25 patients (with $\alpha=5 \%$ and Power $=80 \%$ ).

Results were collected in an excel file and then transferred to SPSS. Fisher's exact test or Mann-Whitney test was used for descriptive statistics. Wilcoxon 
signed Rank Test and student t-test were used for IBS symptoms and severity scores, QOL IBS questionnaire and general health questionnaire, as appropriate. A p-value $<0.05$ was considered significant. All analyses were done using IBM SPSS Statistics for Windows (Version 24.0, IBM Corp., Armonk, NY).

\section{Results}

A total of 22 patients, mean age 54.5, range $22-77$, in a good general health were evaluated. Among them were 16 women and 6 men. 10 patients were first treated with placebo and 12 were given Coltect first. Four were lost to follow-up: One was given only placebo, another one did not receive any treatment, and the other two were treated only with Coltect. The remaining 18 received Coltect and placebo for 4 weeks, each with a 2-week washout interval. Patient characteristics are presented in Table 1. The mean period from diagnosis of IBS was 7.8 \pm 7.6 years. According to Rome III criteria, most (95.5\%) reported gas/abdominal fullness and $63.6 \%$ experienced relief of their symptoms with defecation or different form of stool. Only $10.5 \%$ had mucus secretion.

IBS symptoms: The results are presented in Figure 1. Coltect did not change IBS symptoms, although there was a trend towards better scores in some symptoms. Rating of severity of abdominal gas improved from a mean of $3.47 \pm 1.4$ to $3.21 \pm 1.4, \mathrm{p}=0.36$. Abdominal bloating, diarrhea, constipation and alternating diarrhea and constipation also showed improvement, although not significantly ( 3.47 to $3.42, \mathrm{p}=0.8,2.2$ to $2, \mathrm{p}=0.5,2.2$ to $2, \mathrm{p}=0.2$, and 1.7 to $1.4, \mathrm{p}=0.4$, respectively). The placebo group also reported a similar trend of improvement in abdominal pain, bloating, gas, mucus, and diarrhea. No side effects were reported in the study.

We also compared the differences (deltas) of IBS symptoms between the placebo and Coltect groups (mean scores of placebo before and after treatment minus mean scores of Coltect before and after treatment). The results are presented in Table 2. The deltas did not change significantly, meaning Coltect did not have a better or worse effect on IBS symptoms as compared to placebo.

Severity scores were evaluated using a severity score questionnaire with a scale of 0 - 100, where $0=$ satisfied and $100=$ not satisfied at all. The results are presented in Figure 2. Satisfaction from bowel habits before and after Coltect treatment improved, with a mean score of $70.4 \pm 33.1$ before and $61.8 \pm 29.3$ after Coltect treatment $(\mathrm{p}=0.037)$. However, total severity scores did not differ between the Coltect and placebo groups. The differences (deltas) of the means of severity scores between placebo and Coltect group also did not change (Table 2).

IBS QOL and health related QOL were both evaluated using specific questionnaires as described above. Health-related QOL did not change in either the Coltector placebo group. However, the IBS-QOL scores improved significantly after Coltect treatment, from a mean of $2.5 \pm 0.8$ points to $2.4 \pm 0.7$ points ( $\mathrm{p}=$ 0.036). The change was not statistically significant in the placebo group (Figure $3)$. 


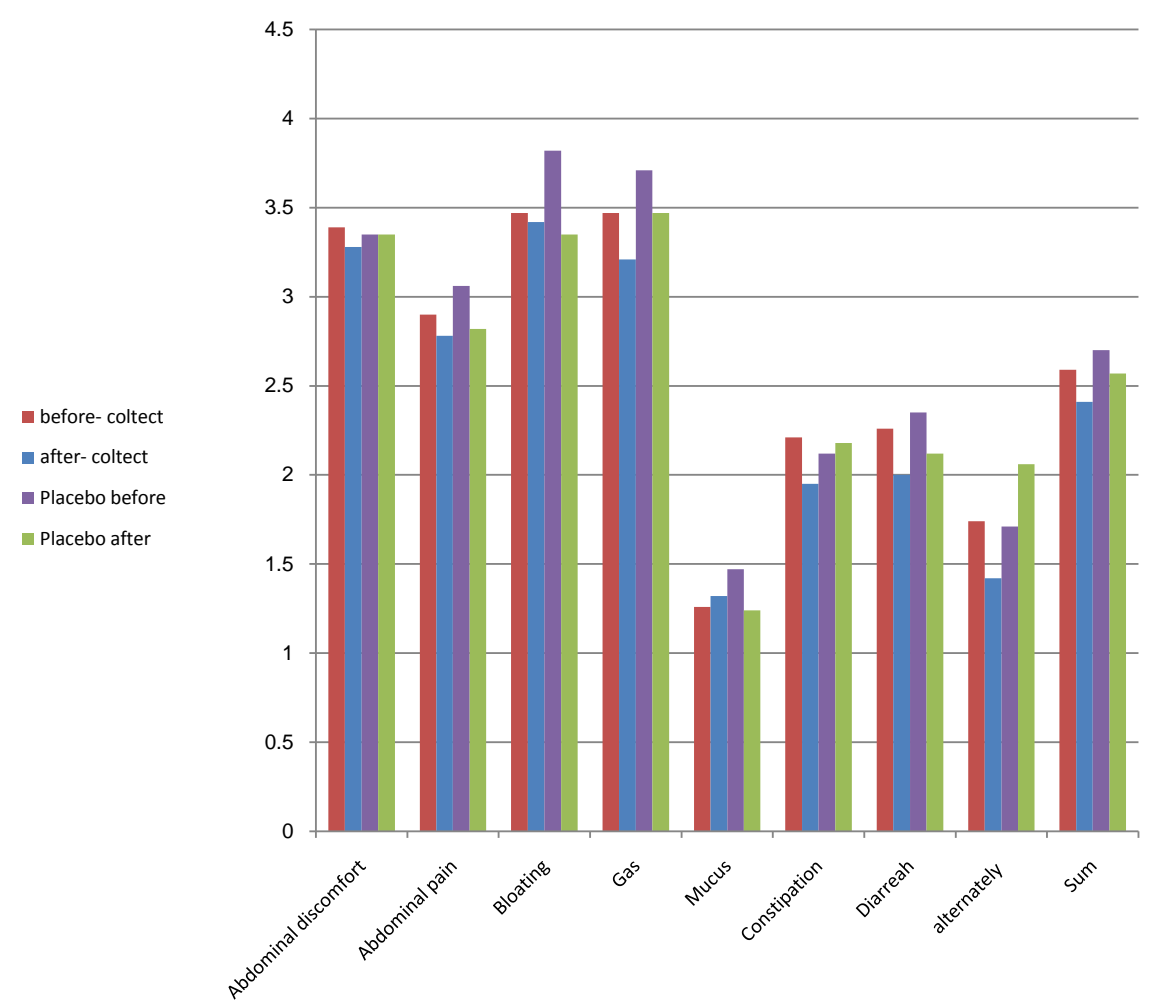

Figure 1. IBS symptoms.

- Coltect before

- Coltect after

- Placebo before

- Placebo after

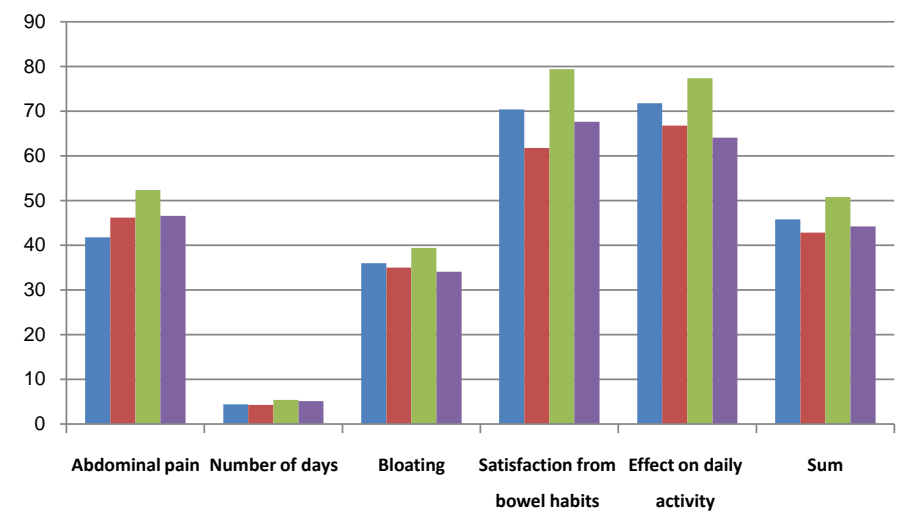

Figure 2. Severity score.

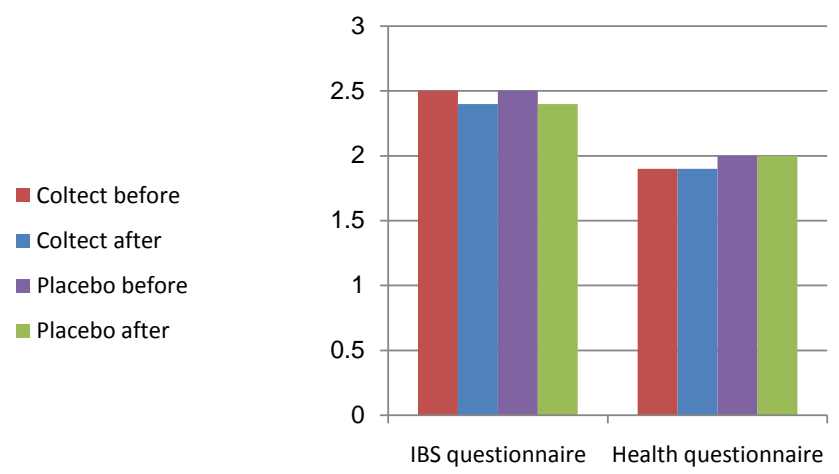

Figure 3. Questionnaires. 
Table 1. Patients' characteristics $(\mathrm{N}=22)$.

\begin{tabular}{cc}
\hline Gender (\%, female) & 72.7 \\
\hline Age (years, mean) & 53.7 \\
BMI (mean) & 22.8 \\
Diagnosis of IBS (years, median) & 7.8 \\
Last episode of IBS (months, median ) & 0 \\
IBS criteria (\%, positive) & \\
Relief with defecation & 63.6 \\
Different frequency of stool & 63.6 \\
Different form of stool & 59.1 \\
Mucus secretion & 9.1 \\
Gas & 95.5 \\
\hline
\end{tabular}

Table 2. Deltas (before-after treatment).

\begin{tabular}{lccccc}
\hline & Coltect & SD & Placebo & SD & P-value \\
\hline $\begin{array}{l}\text { Severity scores, } \\
\mathrm{N}=18 \text { (mean) }\end{array}$ & 0.9 & 52.8 & 23.1 & 128.2 & 0.55 \\
$\begin{array}{l}\text { IBS symptoms, } \\
\mathrm{N}=17 \text { (mean) }\end{array}$ & 0.88 & 5.31 & 1 & 6.75 & 0.96 \\
\hline
\end{tabular}

\section{Discussions}

This prospective, double blind, placebo-controlled, crossover design study evaluated the effect of Coltect on IBS. We found a positive effect of Coltect treatment on satisfaction with changes in bowel habits, a parameter that was evaluated in the severity score questionnaire. Moreover, IBS-related quality of life improved after Coltect treatment, but not after placebo. There was no statistically significant effect of Coltect compared to placebo on other parameters of IBS, including symptoms and general health, although there was a trend towards improvement in abdominal fullness, gas, diarrhea, constipation and alternating bowel movement types.

Evidence supporting the presence of low-grade inflammation in IBS, expressed as increased number of inflammatory cells and metabolites, leading to visceral hypersensitivity and alteration in motility was found in many studies. Törnblom et al. found inflammation and enteric neuropathy in biopsies of the jejunum in IBS patients [8]. Chadwick et al. found an association of activation of mucosal immune system cells in IBS [9]. Therefore, treatment modalities aiming to reduce inflammation may be beneficial in IBS.

In previous studies, curcumin was found to have anti-oxidant activity inhibiting inflammatory mediators [17] and strong anti-inflammatory and anti-oxidant effects in cell cultures [15], animal and human studies [16]. In an animal study, green tea had anti-inflammatory effects on murine colitis [19]. Selenium was 
associated with anti-oxidant and anti-inflammatory effects [20], as well as colorectal cancer prevention. A clinical [21] study with Coltect showed beneficial effects in patients with mild-to-moderate ulcerative colitis [22].

We needed 25 patients to come to a power of $80 \%$, but due to slow recruitment we came to power $(1-\beta)$ of $77 \%$. The gastrointestinal symptoms of these patients referred to IBS only, as other causes were ruled out. Diagnosis of IBS was based on the Rome III criteria and supported by long-term duration of symptoms and lack of other diseases. (Rome III was the standard of evaluation at the time the study was started. Rome IV was published during the study but we thought that changing the eligibility criteria during the study would jeopardize the uniformity of the data). Coltect treatment resulted in improvement in satisfaction from bowel movements and IBS-related quality of life. Coltect treatment seems safe; as no side-effects were reported during the study. Another limitation was the small sample size of 22 participants, only 18 of whom completed follow-up. Therefore, despite the positive indication given by our results, we cannot make conclusions on the effect of Coltect on IBS. Larger studies are needed to evaluate the possible beneficial effects of Coltect on IBS.

\section{Disclosure}

Coltect was provided courtesy of Naveh Pharma, Ltd., Industrial Zone Poleg Netanya, 42505 Israel.

\section{Conflicts of Interest}

The authors declare no conflicts of interest regarding the publication of this paper.

\section{References}

[1] Drossman, D.A., Camilleri, M., Mayer, E.A. and Whitehead, W.E. (2002) AGA Technical Review on Irritable Bowel Syndrome. Gastroenterology, 123, 2108-2131. https://doi.org/10.1053/gast.2002.37095

[2] Peery, A.F., Dellon, E.S., Lund, J., et al. (2012) Burden of Gastrointestinal Disease in the United States: 2012 Update. Gastroenterology, 143, 1179-1187. https://doi.org/10.1053/j.gastro.2012.08.002

[3] Mearin, F., Lacy, B.E., Chang, L., Longstreth, G.F., Thompson, W.G., Chey, W.D., et al. (2006) Functional Bowel Disorders. Gastroenterology, 130, 148-1491.

[4] Ford, A.C., Moayyedi, P., Lacy, B.E., et al. (2014) American College of Gastroenterology Monograph on the Management of Irritable Bowel Syndrome and Chronic Idiopathic Constipation. American Journal of Gastroenterology, 109, S2-S26. https://doi.org/10.1038/ajg.2014.187

[5] Drossman, D.A., Chey, W.D., Johanson, J.F., et al. (2009) Clinical Trial: Lubiprostone in Patients with Constipation-Associated Irritable Bowel Syndrome-Results of Two Randomized, Placebo-Controlled Studies. Alimentary Pharmacology \& Therapeutics, 29, 329-341. https://doi.org/10.1111/j.1365-2036.2008.03881.x

[6] Pimentel, M., Lembo, A., Chey, W.D., et al. (2011) Rifaximin Therapy for Patients with Irritable Bowel Syndrome without Constipation. The New England Journal of 
Medicine, 364, 22-32. https://doi.org/10.1056/NEJMoa1004409

[7] Lembo, A.J., Lacy, B.E., Zuckerman, M.J., et al. (2016) Eluxadoline for Irritable Bowel Syndrome with Diarrhea. The New England Journal of Medicine, 374, 242-253. https://doi.org/10.1056/NEJMoa1505180

[8] Törnblom, H., Lindberg, G., Nyberg, B. and Veress, B. (2002) Full-Thickness Biopsy of the Jejunum Reveals Inflammation and Enteric Neuropathy in Irritable Bowel Syndrome. Gastroenterology, 123, 1972-1979.

[9] Chadwick, V.S., Chen, W., Shu, D., et al. (2002) Activation of the Mucosal Immune System in Irritable Bowel Syndrome. Gastroenterology, 122, 1778-1783.

[10] Guilarte, M., Santos, J., de Torres, I., et al. (2007) Diarrhoea-Predominant IBS Patients Show Mast Cell Activation and Hyperplasia in the Jejunum. Gut, 56, 203-209. https://doi.org/10.1136/gut.2006.100594

[11] Dinan, T.G., Quigley, E.M., Ahmed, S.M., et al. (2006) Hypothalamic-Pituitary-Gut Axis Dysregulation in Irritable Bowel Syndrome: Plasma Cytokines as a Potential Biomarker? Gastroenterology, 130, 304-311.

[12] Bueno, L. (2008) Protease Activated Receptor 2: A New Target for IBS Treatment. European Review for Medical and Pharmacological Sciences, 12, 95-102.

[13] Gecse, K., Róka, R., Ferrier, L., et al. (2008) Increased Faecal Serine Protease Activity in Diarrhoeic IBS Patients: A Colonic Lumenal Factor Impairing Colonic Permeability and Sensitivity. Gut, 57, 591-599. https://doi.org/10.1136/gut.2007.140210

[14] Liebregts, T., Adam, B., Bredack, C., et al. (2007) Immune Activation in Patients with Irritable Bowel Syndrome. Gastroenterology, 132, 913-920.

[15] Larmonier, C.B., Midura-Kiela, M.T., Ramalingam, R., et al. (2011) Modulation of Neutrophil Motility by Curcumin: Implications for Inflammatory Bowel Disease. Inflammatory Bowel Diseases, 17, 503-515. https://doi.org/10.1002/ibd.21391

[16] Epstein, J., Docena, G., MacDonald, T.T. and Sanderson, I.R. (2010) Curcumin Suppresses p38 Mitogen-Activated Protein Kinase Activation, reduces IL- $1 \beta$ and Matrix Metalloproteinase-3 and Enhances IL-10 in the Mucosa of Children and Adults with Inflammatory Bowel Disease. British Journal of Nutrition, 103, 824-832. https://doi.org/10.1017/S0007114509992510

[17] Jobin, C., Bradham, C.A., Russo, M.P., et al. (1999) Curcumin Blocks Cytokine Mediated NF-Kappa B Activation and Proinflammatory Gene Expression by Inhibiting Inhibitory Factor I-Kappa B Kinase Activity. The Journal of Immunology, 163, 3474-3483.

[18] Gupta, S.C., Patchva, S. and Aggarwal, B.B. (2013) Therapeutic Roles of Curcumin: Lessons Learned from Clinical Trials. The AAPS Journal, 15, 195-218. https://doi.org/10.1208/s12248-012-9432-8

[19] Mazzon, E., Muià, C., Paola, R.D., Genovese, T., Menegazzi, M., De Sarro, A., Suzuki, H. and Cuzzocrea, S. (2005) Green Tea Polyphenol Extract Attenuates Colon Injury Induced by Experimental Colitis. Free Radical Research, 39, 1017-1025. https://doi.org/10.1080/10715760500197177

[20] Sunde, R.A. (2010) Selenium. In: Coates, P.M., Betz, J.M., Blackman, M.R., et al., Eds., Encyclopedia of Dietary Supplements, 2nd Edition, Informa Healthcare, London and New York, 711-718.

[21] Thompson, P.A., Ashbeck, E.L., Roe, D.J., Fales, L., Buckmeier, J., Wang, F., Bhattacharyya, A., Hsu, C.H., Chow, H.H., Ahnen, D.J., Boland, C.R., Heigh, R.I., Fay, D.E., Hamilton, S.R., Jacobs, E.T., Martinez, M.E. and Alberts, D.S. (2016) Selenium Supplementation for Prevention of Colorectal Adenomas and Risk of Associated Type 2 Diabetes. Journal of the National Cancer Institute, 108. 
https://doi.org/10.1093/jnci/djw152

[22] Shapira, S., Lesho, A., Katz, D., et al. (2017) Of Mice and Men: A Novel Dietary Supplement for a Treatment of Ulcerative Colitis. Therapeutic Advances in Gastroenterology, 11. https://doi.org/10.1177/1756283X17741864

[23] Altman, D.G. and Bland, J.M. (1999) How to Randomise. British Medical Journal, 319, 703-704. https://doi.org/10.1136/bmj.319.7211.703

[24] Drossman, D.A., Patrick, D.L., Whitehead, W.E., Toner, B.B., Diamant, N.E., Hu, Y., Jia, H. and Bangdiwala, S.I. (2000) Further Validation of the IBS-QOL: A Disease-Specific Quality-of-Life Questionnaire. American Journal of Gastroenterology, 95, 1000-1007.

[25] Drossman, D., Morris, C.B., Hu, Y., Toner, B.B., Diamant, N., Whitehead, W.E., Dalton, C.B., Leserman, J., Patrick, D.L. and Bangdiwala, S.I. (2007) Characterization of Health Related Quality of Life (HRQOL) for Patients With Functional Bowel Disorder (FBD) and Its Response to Treatment. American Journal of Gastroenterology, 102, 1442-1453. 